\title{
Glans Ischemia after Circumcision in a 16-Year- Old Boy: Full Recovery after Angiography with Local Spasmolysis, Systemic Vasodilatation, and Anticoagulation
}

\author{
Richard Gnatzy, MD ${ }^{1}$ Jochen Fuchs, MD² Manuela Siekmeyer, MD ${ }^{3}$ Anne Bettina Beeskow, MD \\ Jan-Hendrik Gosemann, $\mathrm{MD}^{1}$ Martin Lacher, MD, $\mathrm{PhD}^{1}$ \\ ${ }^{1}$ Department of Pediatric Surgery, University of Leipzig, Leipzig, \\ Germany \\ 2 Department of Diagnostic and Interventional Radiology, 310 Klinik \\ $\mathrm{GmbH}$, Nuremberg, Germany \\ ${ }^{3}$ Hospital for Children and Adolescents, University of Leipzig, Leipzig, \\ Germany \\ ${ }^{4}$ Department of Radiology, University of Leipzig, Leipzig, \\ Germany \\ Address for correspondence Richard Gnatzy, MD, Department of \\ Pediatric Surgery, University of Leipzig, Liebigstrasse 20a, Leipzig 04103, \\ Germany (e-mail: richard.gnatzy@medizin.uni-leipzig.de).
}

Eur J Pediatr Surg Rep 2018;6:e66-e69.

\begin{abstract}
Circumcision is one of the most frequent procedures performed by pediatric surgeons. A dorsal penile nerve block (DPNB) is commonly used for perioperative analgesia. We report the case of a 16-year-old boy with ischemia of the glans who presented on the first postoperative day after circumcision under DPNB ( $0.25 \%$ bupivacaine) at an outside hospital. The patient underwent immediate angiography under sedation. An

Keywords

- Glans Ischemia

- children

- digital subtraction angiography intra-arterial spasmolysis was performed with alprostadil and nitroglycerine and a sufficient perfusion of the glans penis was confirmed. Subsequently, systemic sildenafil, arginine, and heparin were given. Following this 3-day medical treatment, ischemia resolved completely. Our case emphasizes the role of invasive angiography in the diagnostic workup and the therapeutic possibilities of local spasmolysis, systemic vasodilatation, and anticoagulation.
\end{abstract}

\section{Introduction}

Circumcision is one of the most frequent procedures performed by pediatric surgeons. However, after surgery some patients represent to the emergency department due to bleeding, pain, swelling, redness, local infection, and decreased urinary output. Most of this morbidity is minor and can be easily managed. ${ }^{1}$ In contrast, ischemia or even necrosis of the glans is a rare complication; in the past 15 years, nine pediatric cases have been reported in the literature. $^{2-8}$ We report the case of a boy with ischemia of the glans penis after circumcision at an outside hospital.

received

June 10, 2018

accepted after revision

June 23, 2018
DOI https://doi.org/

10.1055/s-0038-1667330.

ISSN 2194-7619.

\section{Case Report}

A 16-year-old boy with phimosis underwent elective circumcision at an outside hospital. Anesthesia was performed via dorsal penile nerve block (DPNB) ( $15 \mathrm{~mL}$ of $0.25 \%$ bupivacaine). On the first postoperative day, he was referred to our hospital due to pain, black discoloration, and swelling of the glans. Voiding was possible. On clinical examination, the distal glans showed severe ischemia ( - Fig. 1). All laboratory results including blood count, lactate, D-dimer, and clotting profile were within normal limits. Color Doppler ultrasound of the penis showed good cavernosal artery flow to the glans. After

\footnotetext{
(c) 2018 Georg Thieme Verlag KG Stuttgart · New York
}

License terms

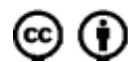




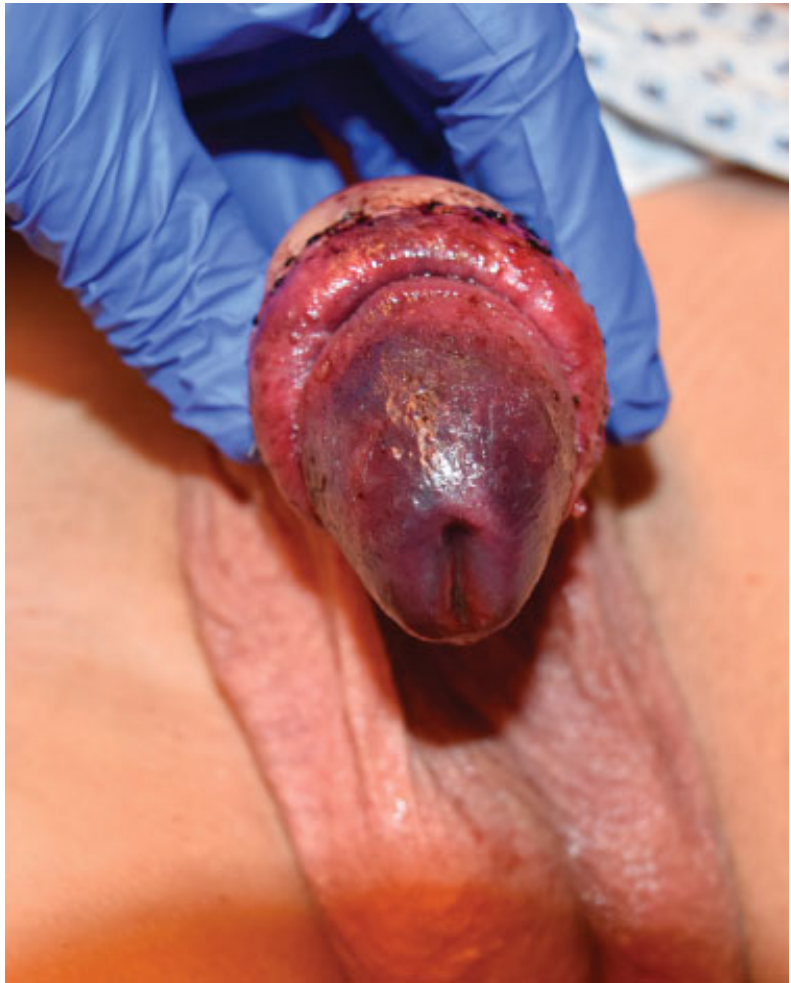

Fig. 1 Glans with severe ischemia on the first postoperative day (admission).

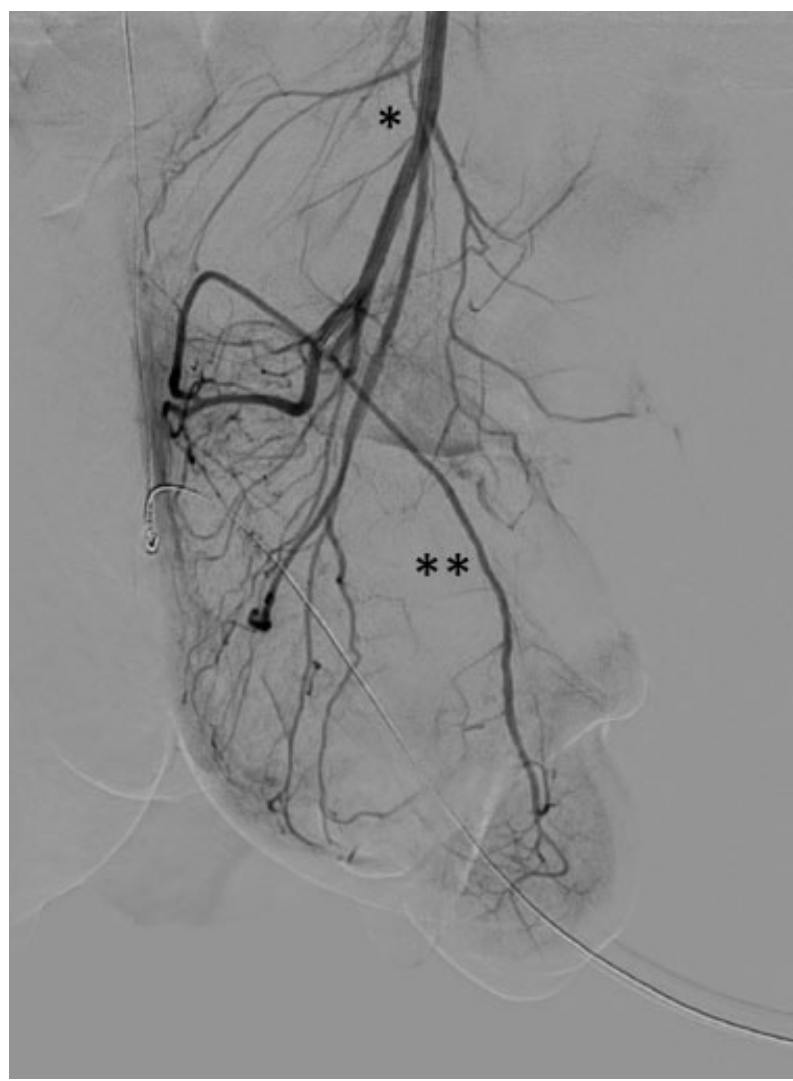

Fig. 2 Digital subtraction angiography (DSA) of the left internal pudendal artery $\left(^{*}\right)$ via microcatheter (Progreat 2.7F, Terumo) confirmed a sufficient arterial perfusion of the glans with good contrast filling of the dorsal artery of the penis $\left({ }^{* *}\right)$.

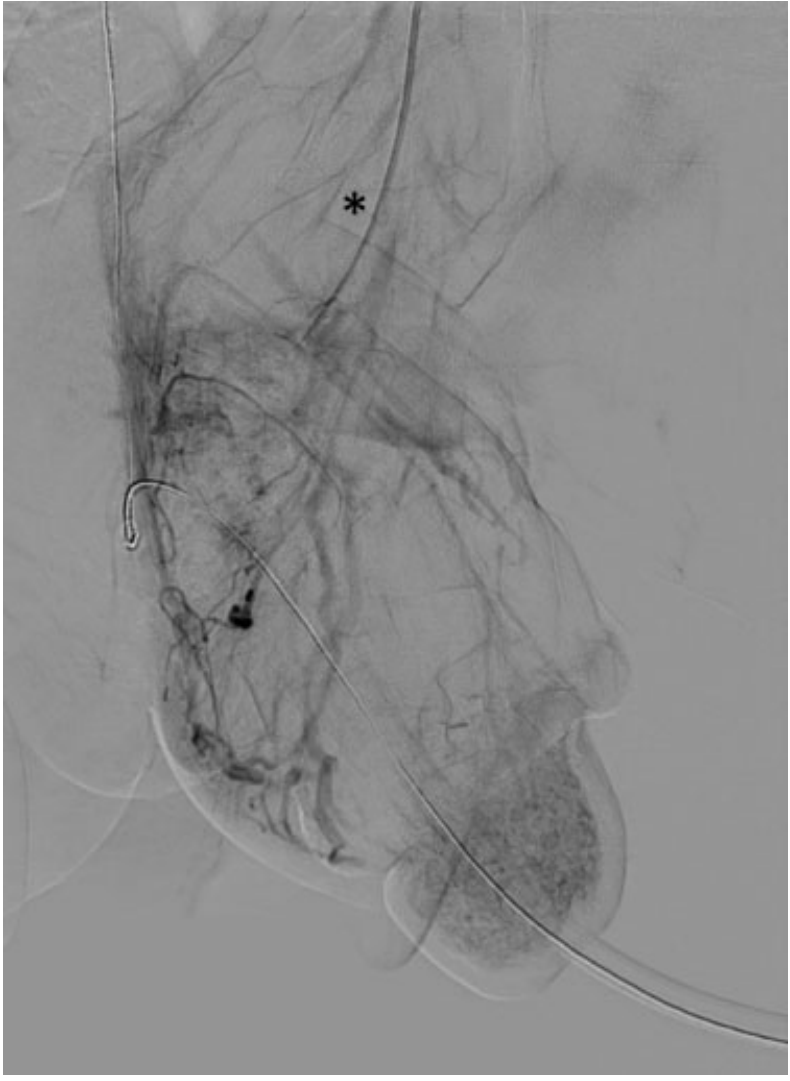

Fig. 3 Venous phase of the digital subtraction angiography (DSA) of the left internal pudendal artery $\left({ }^{*}\right)$ showed a sufficient venous drainage of the glans.

transferral to our pediatric intensive care unit, a caudal block was performed to reduce sympathetic tone and improve arterial blood flow. Five hours after admission, the patient underwent digital subtraction angiography (DSA) under sedation. After overwiew of the pelvic arteries and the left internal iliac artery, the internal pudendal artery was explored selectively via microcatheter (Progreat 2.7F, Terumo) but no vasospasm or thrombus was detected. A sufficient arterial perfusion as well as normal venous drainage of the glans was confirmed ( - Fig. 2 and 3 ). To use all therapeutic options, intraarterial spasmolysis with a bolus of $5 \mu \mathrm{g}$ alprostadil and $150 \mu \mathrm{g}$ nitroglycerine was sequentially given. Eight hours after admission, systemic therapy with sildenafil $(1 \mathrm{mg} / \mathrm{kg}$ orally once a day), L-arginine-hydrochlorid $(0.1 \mathrm{mg} / \mathrm{kg} / \mathrm{hour})$, and unfractionated heparin (15 units/kg/hour, up to 20 units/ $\mathrm{kg} /$ hour depending on partial thromboplastin time) were initiated and given for 3 days.

After 3 days of systemic vasodilatative therapy, the darkish color of the glans changed to a brownish appearance. A surgical intervention was not necessary and ischemia resolved completely. The boy was discharged on the seventh postoperative day without adverse events ( - Fig. 4).

\section{Discussion}

Glans ischemia is an extremely rare complication after circumcision. In most cases, the etiology remains unclear. Apart from hematoma, tight suture lines, or excessive use of 


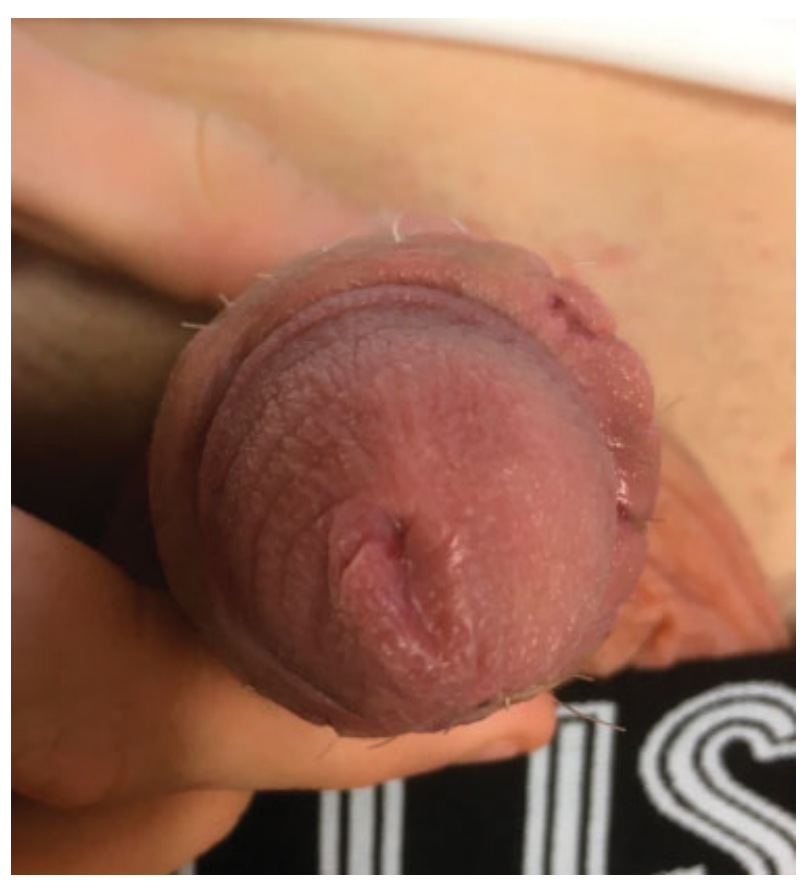

Fig. 4 After treatment, glans ischemia resolved completely (seventh postoperative day after angiography).

monopolar cautery, DPNB has been suggested to be the most frequent cause of this complication. ${ }^{4,9}$ In a large cohort from Singapore analyzing 3,909 DPNB, the total complication rate was reported to be as low as $0.23 \%{ }^{10}$ Other authors also report on ischemia of the glans penis following DPNB for circumcision. $^{6,7,11}$ As an alternative for DPNB, topical anesthetics have been evaluated but did not provide sufficient perioperative analgesia. ${ }^{12}$ Kaplanian et al suggested to limit the volume of local anesthetic solution to an amount of $0.2 \mathrm{~mL} / \mathrm{kg}$ (up to maximum of $10 \mathrm{~mL}$ ). ${ }^{7}$ In our patient, the primary surgeons exceeded this dose and applied $15 \mathrm{~mL}$ of plain bupivacaine on each side. Therefore, this could have led to a transient vasospasm.

In case of glans ischemia following DPNB, the main goal of treatment is the reestablishment of sufficient blood flow to the penis. Several therapeutic approaches have been described including pentoxifylline, hyperbaric therapy, iloprost, enoxaparin, anticoagulation, local testosterone, and peridural anesthesia. ${ }^{2-8}$

In our case, we started treatment by performing a caudal epidural block to improve arterial supply and to reduce sympathetic tone and pain. This is in line with a report of a group from Australia, who successfully applied this technique in a 9-year-old boy. ${ }^{7}$

Thereafter, we focused on treating the suspected vascular origin of glans ischemia. In adult urologic departments, DSA is frequently used in the diagnostic workup for arteriogenic impotence and percutaneous endovascular revascularization to treat vasculogenic erectile dysfunction (penile artery stenosis $)^{13-15}$ or in the treatment of high-flow priapism. ${ }^{16}$ To the best of our knowledge, we applied invasive angiography for the first time in a patient with glans ischemia. We observed a sufficient arterial perfusion as well as a normal venous drainage of the glans. A vasospasm or arterial obstruction, as well as a venous outflow problem, was ruled out during this procedure. Additionally, potent vasodilators nitroglycerine (nitric oxide as cyclic guanosine monophosphate activator) and alprostadil (prostaglandin E1 analog) were applied intra-arterially to test if this could further improve the perfusion, which was not the case.

The nonselective phosphodiesterase inhibitor pentoxifylline (PTX) is a hemorheological drug, which improves peripheral blood flow by reducing whole blood viscosity. It works by relaxing smooth muscle of the corpus cavernosum. Two case reports on a 3-and 10-year-old boy combined the therapy of caudal block with PTX and described good outcomes. ${ }^{4,6}$ However, indication and dosage of PTX in children remain controversial. ${ }^{3,4,6,8,11}$ Hence, in our patient we used the selective phosphodiesterase inhibitor (PDE 5) sildenafil as a vasodilative agent with success and did not appreciate side effects.

Efe et al suggested a monotherapy with low molecular weight heparin (enoxaparin) as the treatment of choice for ischemia of the glans in a 7-year-old boy. After 5 days of anticoagulation, the black discoloration of the glans disappeared. ${ }^{5}$ In our patient, we used the protocol of unfractionated heparin (15-20 units/kg/hour) according to the protocol of Sara and Lowry ([bupivacaine 0.5\%] with lowdose heparin infusion [25 units/kg/hour] for 4 days).$^{9}$

Other therapeutic options include hyperbaric therapy, which has been evaluated in adults only. ${ }^{17}$ Therefore, we did not consider this therapeutic option.

\section{Conclusion}

Ischemia of the glans is a rare complication after circumcision after DPNB. Although the cause of the transient ischemia could not be proven, we speculate that DPNB might have caused severe vasospasm. In the current patient, the multimodal treatment resulted in complete recovery of the glans without adverse events. Our case emphasizes the role of DSA in the diagnostic workup and the therapeutic possibilities of local spasmolysis, systemic vasodilatation, and anticoagulation.

We acknowledge support from the German Research Foundation (DFG) and Leipzig University within the program of Open Access Publishing.

\section{Conflict of Interest}

None.

\section{References}

1 Gold G, Young S, O'Brien M, Babl FE. Complications following circumcision: presentations to the emergency department. J Paediatr Child Health 2015;51(12):1158-1163

2 Aminsharifi A, Afsar F, Tourchi A. Delayed glans necrosis after circumcision: role of testosterone in salvaging glans. Indian J Pediatr 2013;80(09):791-793

3 Aslan A, Karagüzel G, Melikoglu M. Severe ischemia of the glans penis following circumcision: a successful treatment via pentoxifylline. Int J Urol 2005;12(07):705-707

4 Cárdenas Elías MÁ, Vázquez Rueda F, Jiménez Crespo V, et al. An unexpected complication: glans ischemia after circumcision. Review of the literature [in Spanish]. Cir Pediatr 2016;29(03):127-130 
5 Efe E, Resim S, Bulut BB, et al. Successful treatment with enoxaparin of glans ischemia due to local anesthesia after circumcision. Pediatrics 2013;131(02):e608-e611

6 Garrido-Abad P, Suárez-Fonseca C. Glans ischemia after circumcision and dorsal penile nerve block: case report and review of the literature. Urol Ann 2015;7(04):541-543

7 Kaplanian S, Chambers NA, Forsyth I. Caudal anaesthesia as a treatment for penile ischaemia following circumcision. Anaesthesia 2007;62(07):741-743

8 Karaguzel E, Tok DS, Kazaz IO, et al. Postcircumcisional ischemia of the glans penis treated with pentoxifylline. Case Rep Urol 2013; 2013:278523

9 Sara CA, Lowry CJ. A complication of circumcision and dorsal nerve block of the penis. Anaesth Intensive Care 1985;13(01): 79-82

10 Soh CR, Ng SB, Lim SL. Dorsal penile nerve block. Paediatr Anaesth 2003;13(04):329-333

11 Migliorini F, Bianconi F, Bizzotto L, Porcaro AB, Artibani W. Acute ischemia of the glans penis after circumcision treated with hyperbaric therapy and pentoxifylline: case report and revision of the literature. Urol Int 2018;100(03):361-363
12 Salgado Filho MF, Gonçalves HB, Pimentel Filho LH, et al. Assessment of pain and hemodynamic response in older children undergoing circumcision: comparison of eutectic lidocaine/prilocaine cream and dorsal penile nerve block. J Pediatr Urol 2013;9 (05):638-642

13 Goldstein I, Bastuba M, Lurie A, Lubisich J. Penile revascularization. J Sex Med 2008;5(09):2018-2021

14 Spiliopoulos S, Shaida N, Katsanos K, Krokidis M. The role of interventional radiology in the diagnosis and management of male impotence. Cardiovasc Intervent Radiol 2013;36(05): 1204-1212

15 Wang TD, Lee WJ, Yang SC, et al. Clinical and imaging outcomes up to 1 year following balloon angioplasty for isolated penile artery stenoses in patients with erectile dysfunction: the PERFECT-2 study. J Endovasc Ther 2016;23(06):867-877

16 Fuchs J, Stumpp P, Moche M. Posttraumatic high flow priapism: successful treatment with superselective embolization [in German]. RoFo Fortschr Geb Rontgenstr Nuklearmed 2012;184(02): 147-148

17 Mitchell SJ, Bennett MH. Unestablished indications for hyperbaric oxygen therapy. Diving Hyperb Med 2014;44(04):228-234 